\title{
Assessment of Hyposalivation, Xerostomia, and Oral Health-Related Quality of Life in Polymedicated Patients ${ }^{\dagger}$
}

\author{
João Gato Marques ${ }^{1, *(D)}$, Cecília Rozan ${ }^{1}\left(\mathbb{D}\right.$, Luís Proença ${ }^{1,2} \mathbb{D}$, André Peixoto ${ }^{1}$ and Cristina Manso ${ }^{1,2}$ \\ 1 Instituto Universitário Egas Moniz, Campus Universitário, 2829-511 Caparica, Portugal; \\ ceciliarozan@gmail.com (C.R.); lproenca@egasmoniz.edu.pt (L.P.); andrec.peixoto@gmail.com (A.P.); \\ mansocristina@gmail.com (C.M.) \\ 2 Centro de Investigação Interdisciplinar Egas Moniz, 2829-511 Caparica, Portugal \\ * Correspondence: jgato1997@gmail.com \\ + Presented at the 5th International Congress of CiiEM-Reducing Inequalities in Health and Society, Online, \\ 16-18 June 2021
}

check for updates

Citation: Marques, J.G.; Rozan, C.; Proença, L.; Peixoto, A.; Manso, C. Assessment of Hyposalivation, Xerostomia, and Oral Health-Related Quality of Life in Polymedicated Patients. Med. Sci. Forum 2021, 5, 6. https://doi.org/10.3390/msf 2021005006

Academic Editors: Helena Barroso and Cidália Castro

Published: 11 July 2021

Publisher's Note: MDPI stays neutral with regard to jurisdictional claims in published maps and institutional affiliations.

Copyright: (c) 2021 by the authors. Licensee MDPI, Basel, Switzerland. This article is an open access article distributed under the terms and conditions of the Creative Commons Attribution (CC BY) license (https:// creativecommons.org/licenses/by/ $4.0 /)$.

\begin{abstract}
To investigate the self-reported impact of hyposalivation/xerostomia on Oral HealthRelated Quality of Life (OHRQoL) reported by polymedicated patients and evaluate the association between hyposalivation/xerostomia and OHRQoL. A cross-sectional observational study was performed on 40 polymedicated patients selected from the Clínica Dentária Egas Moniz. The subjects signed a consent form, answered a questionnaire to assess xerostomia via the Summated Xerostomia Inventory (SXI-PL) and The Portuguese short version of the Oral Health Impact Profile Questionnaire (OHIP$14 \mathrm{sp})$, and underwent sialometry evaluation. Patients with hyposalivation showed higher scores of SXI-PL (8.60 \pm 2.56$)$ and OHIP-14sp (16.0 \pm 15.8$)$. These findings suggest an association between hyposalivation and SXI-PL and OHIP-14sp scores $(p<0.05)$.
\end{abstract}

Keywords: hyposalivation; xerostomia; oral health-related quality of life; polymedication

\section{Introduction}

Saliva is a biochemically complex fluid containing proteins and various glycoproteins, lipids, electrolytes, and buffers, which all play a vital role in oral health. It preserves dentition, inhibits the growth of microorganisms, lubricates and protects the oral mucosa from trauma, and facilitates mastication, deglutition, and speech. Hyposalivation and xerostomia are the most common symptoms associated with polymedication. Consequently, the oral signs and symptoms of hyposalivation and xerostomia extend far beyond those of simple dryness, with a negative impact on patients' quality of life [1]. The aim of this study was to assess the prevalence of hyposalivation and xerostomia in polymedicated patients and their impact on Oral Health-Related Quality of Life (OHRQoL).

\section{Materials and Methods}

We performed a cross-sectional observational study. We selected 40 polymedicated patients, who were regularly administered at least two of the following medications: anticonvulsants, antidepressants, oral hypoglycemics, antihypertensives, and H1 antihistamines, from the Clínica Dentária Egas Moniz. The study was approved by the Egas Moniz Ethical Committee. The subjects signed a consent form and answered a questionnaire to assess Summated Xerostomia Inventory (SXI-PL) and The Portuguese short version of the Oral Health Impact Profile Questionnaire (OHIP-14sp) and underwent sialometry evaluation to assess unstimulated (USFR) and stimulated (SSFR) salivary flow rates. Hyposalivation was diagnosed for USFR $<0.1 \mathrm{~mL} / \mathrm{min}$ and/or SSFR $<0.7 \mathrm{~mL} / \mathrm{min}$. A statistical analysis was performed using the software IBM SPSS ${ }^{\circledR}$ Statistics, v.25. Spearman's correlation coefficient was used to analyze the association of the SXI-PL and OHIP-14sp scores with salivary flow rates. 


\section{Results and Discussion}

The prevalence of hyposalivation was $50 \%$ in our population. The overall SXI-PL scores ranged from 5 to 15 with a mean of $7.85( \pm 2.37)$. The mean score of SXI-PL was higher in patients with hyposalivation $(8.60 \pm 2.56)$. The results demonstrate a statistically significant correlation between SXI-PL scores and hyposalivation $(p=0.0195)$ (Table 1$)$. The symptom that indicated higher severity of xerostomia was "My mouth feels dry" (2.20 \pm 0.70 ) (Figure 1a).

Table 1. Discriminant validity of Summated Xerostomia Inventory (SXI-PL) and The Portuguese short version of the Oral Health Impact Profile Questionnaire (OHIP-14sp) mean scores for assessing salivary flow conditions.

\begin{tabular}{ccccc}
\hline & Hyposalivation & Normal & Total & $p$-Value ${ }^{\mathbf{1}}$ \\
\hline SXI-PL & $8.60 \pm 2.56$ & $7.10 \pm 1.94$ & $7.85 \pm 2.37$ & 0.0195 \\
OHIP-14sp & $16.0 \pm 15.8$ & $7.0 \pm 7.4$ & $11.50 \pm 12.98$ & 0.0425 \\
\hline
\end{tabular}

${ }^{1}$ A significance level of 0.05 was considered statistically significant.

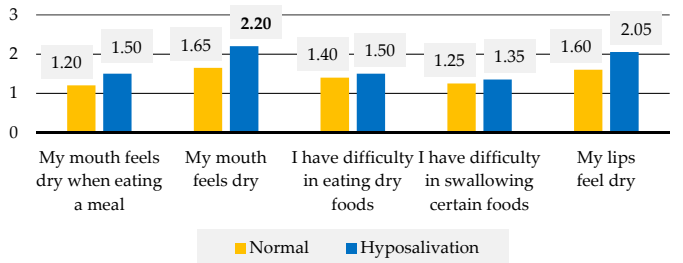

(a)

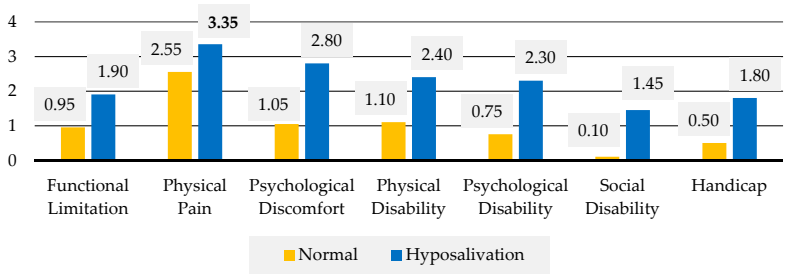

(b)

Figure 1. (a) Distribution of the mean scores of SXI-PL symptoms for different salivary flow condition; (b) distribution of the mean scores of OHIP-14sp dimensions for different salivary flow condition.

The overall OHIP-14sp scores ranged from 0 to 51 , with a mean of $11.50( \pm 12.98)$. The mean score of OHIP-14sp was higher in patients with hyposalivation (16.0 \pm 15.8$)$. The results demonstrate a statistically significant correlation between OHIP-14sp scores and hyposalivation $(p=0.0425)$ (Table 1$)$. The dimension of OHIP-14sp with higher impact was "Physical Pain" (3.35 \pm 2.81$)$ (Figure 1b).

Munõz et al. determined that xerostomia was prevalent in $55.9 \%$ of an elderly population with hyposalivation in Chile [1]. Putten et al. reported that the most severe symptom of xerostomia in a Dutch population was "My mouth feels dry" (1.8 points) [2]. Smidt et al. found an association between hyposalivation and xerostomia in Copenhagen inhabitants $(p<0.001)[3]$.

Ikebe et al. performed a study of hyposalivation and its impact on OHRQoL in an elderly Japanese population. The results demonstrated that subjects with hyposalivation reported higher OHIP-14 scores (14.6 \pm 8.3) and showed a higher impact on the "Physical Pain" dimension (1.5 points). An association between hyposalivation and OHIP-14 was found $(p=0.011)[4]$.

In the present study, an association between hyposalivation and xerostomia was found in polymedicated patients, which has a negative impact on oral health-related quality of life.

Conflicts of Interest: The authors declare no conflict of interest.

\section{References}

1. Munõz, C.; Martínez, A.; Flores, M.; Catalán, A. Relationship between Xerostomia and Hyposalivation in Senior Chilean People. Rev. Clin. Periodoncia Implantol. Rehabil. Oral 2019, 12, 123-126. [CrossRef]

2. Putten, G.J.; Brand, H.S.; Schols, J.M.; Baat, C. The diagnostic suitability of a xerostomia questionnaire and the association between xerostomia, hyposalivation and medication use in a group of nursing home residents. Clin. Oral Investig. 2011, 15, 185-192. [CrossRef] [PubMed] 
3. Smidt, D.; Torpet, L.A.; Nauntofte, B.; Heegaard, K.M.; Pedersen, A.M. Associations between oral and ocular dryness, labial and whole salivary flow rates, systemic diseases and medications in a sample of older people. Community Dent. Oral Epidemiol. 2011, 39, 276-288. [CrossRef] [PubMed]

4. Ikebe, K.; Matsuda, K.; Morii, K.; Wada, M.; Hazeyama, T.; Nokubi, T.; Ettinger, R.L. Impact of dry mouth and hyposalivation on oral health-related quality of life of elderly Japanese. Oral Surg. Oral Med. Oral Pathol. Oral Radiol. Endod. 2007, 103, $216-222$. [CrossRef] [PubMed] 